Article

\title{
Generation and Application of the Zebrafish heg1 Mutant as a Cardiovascular Disease Model
}

\author{
Shuxian Lu, Mengyan Hu, Zhihao Wang, Hongkai Liu, Yao Kou, Zhaojie Lyu and Jing Tian *๑ \\ Key Laboratory of Resource Biology and Biotechnology in Western China, Ministry of Education, \\ School of Medicine, Northwest University, Xi'an 710069, China; lushuxian@stumail.nwu.edu.cn (S.L.); \\ humengyan@stumail.nwu.edu.cn (M.H.); zhihaowang9264@gmail.com (Z.W.); \\ liuhongkai@stumail.nwu.edu.cn (H.L.); kouyao@stumail.nwu.edu.cn (Y.K.); \\ lyuzhaojie@stumail.nwu.edu.cn (Z.L.) \\ * Correspondence: tianjing@nwu.edu.cn; Tel.: +86-29-88302339
}

Received: 11 October 2020; Accepted: 10 November 2020; Published: 12 November 2020

\begin{abstract}
Cardiovascular disease (CVD) is the leading cause of global mortality, which has caused a huge burden on the quality of human life. Therefore, experimental animal models of CVD have become essential tools for analyzing the pathogenesis, developing drug screening, and testing potential therapeutic strategies. In recent decades, zebrafish has entered the field of CVD as an important model organism. HEG1, a heart development protein with EGF like domains 1, plays important roles in the development of vertebrate cardiovascular system. Loss of HEG1 will affect the stabilization of vascular endothelial cell connection and eventually lead to dilated cardiomyopathy (DCM). Here, we generated a heg1-specific knockout zebrafish line using CRISPR/Cas9 technology. Zebrafish heg1 mutant demonstrated severe cardiovascular malformations, including atrial ventricular enlargement, heart rate slowing, venous thrombosis and slow blood flow, which were similar to human heart failure and thrombosis phenotype. In addition, the expression of zebrafish cardiac and vascular markers was abnormal in heg1 mutants. In order to apply zebrafish heg1 mutant in cardiovascular drug screening, four Traditional Chinese Medicine (TCM) herbs and three Chinese herbal monomers were used to treat heg1 mutant. The pericardial area, the distance between sinus venosus and bulbus arteriosus (SV-BA), heart rate, red blood cells (RBCs) accumulation in posterior cardinal vein (PCV), and blood circulation in the tail vein were measured to evaluate the therapeutic effects of those drugs on DCM and thrombosis. Here, a new zebrafish model of DCM and thrombosis was established, which was verified to be suitable for drug screening of cardiovascular diseases. It provided an alternative method for traditional in vitro screening, and produced potential clinical related drugs in a rapid and cost-effective way.
\end{abstract}

Keywords: cardiovascular disease (CVD); dilated cardiomyopathy (DCM); thrombosis; zebrafish model; CRISPR/Cas9; heg1; Traditional Chinese Medicine (TCM); drug screening

\section{Introduction}

The cardiovascular system is the first organ system to form and function in vertebrate embryos. Cardiovascular disease (CVD) generally refers to the heart ischemic or hemorrhagic diseases caused by hyperlipidemia, blood viscosity, atherosclerosis, and hypertension, etc. [1]. An article published in The Lancet in 2020 investigated cardiovascular disease admissions and deaths among middle-aged adults in 21 countries. The results showed that cardiovascular disease was the most common cause of hospitalization (23.9\%) and the most common cause of death (40\%) [2]. Therefore, the development of effective and safe therapeutic drugs for CVD has proven to be challenging. 
Zebrafish, a useful tool to study human diseases, is an excellent model for cardiovascular disease research. Zebrafish embryonic heart has two chambers, atrium and ventricle. Heart development in zebrafish starts very early; cardiac progenitors can be found at the blastula stage, about $5 \mathrm{~h}$ after fertilization (hpf). At $24 \mathrm{hpf}$, the heart tube forms, and leads to the ventricle to be anterior and the atrium to be posterior. The coordinated atrial and ventricular contraction develops at $36 \mathrm{hpf}$ (Figure 1A) [3]. Venous blood flows into atrium then into the ventricle through the one-way atrioventricular valve. The ventricle then pumps blood to the body through a one-way outflow valve. The general pattern of the zebrafish heart formation is similar to that of early mammalians. Therefore, the heart of zebrafish at $48 \mathrm{hpf}$ is comparable in morphology and physiology to the heart of a 12-day mouse or 35-day human embryo [4]. Zebrafish hearts have coronary blood vessels, and their heart rate (HR) (120/140 beats per minute) is closer to humans (60/100 beats per minute) than mice (300/600 beats per minute) [5]. One notable exception to zebrafish blood circulation is that, during the first week after fertilization, embryos do not rely on convective circulation, because they obtain sufficient oxygen through diffusion. This allows the embryos with cardiovascular defects to continue to develop until they outgrow the diffusion distance of oxygen. This facilitates more extensive phenotypic assessment of mutations, which in mammals can lead to very early embryonic lethality [6,7]. In addition, zebrafish thrombocytes are homologous to mammalian platelets, and the hemostatic mechanisms of zebrafish cells and body fluids are similar to those of human beings, which indicates that zebrafish can be used as a model for hemostasis and thrombosis in mammals [8,9]. Moreover, zebrafish transparent embryos make it easy to observe phenotypic changes, which make zebrafish a rapid, simple and low-cost cardiovascular drug-screening system [10].

A
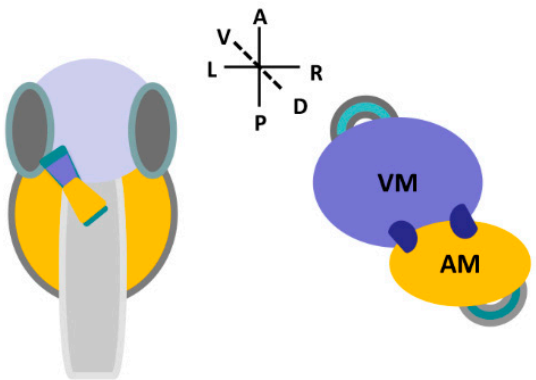

VM: Ventricle Myocardium Endocardium Sinus Venosus
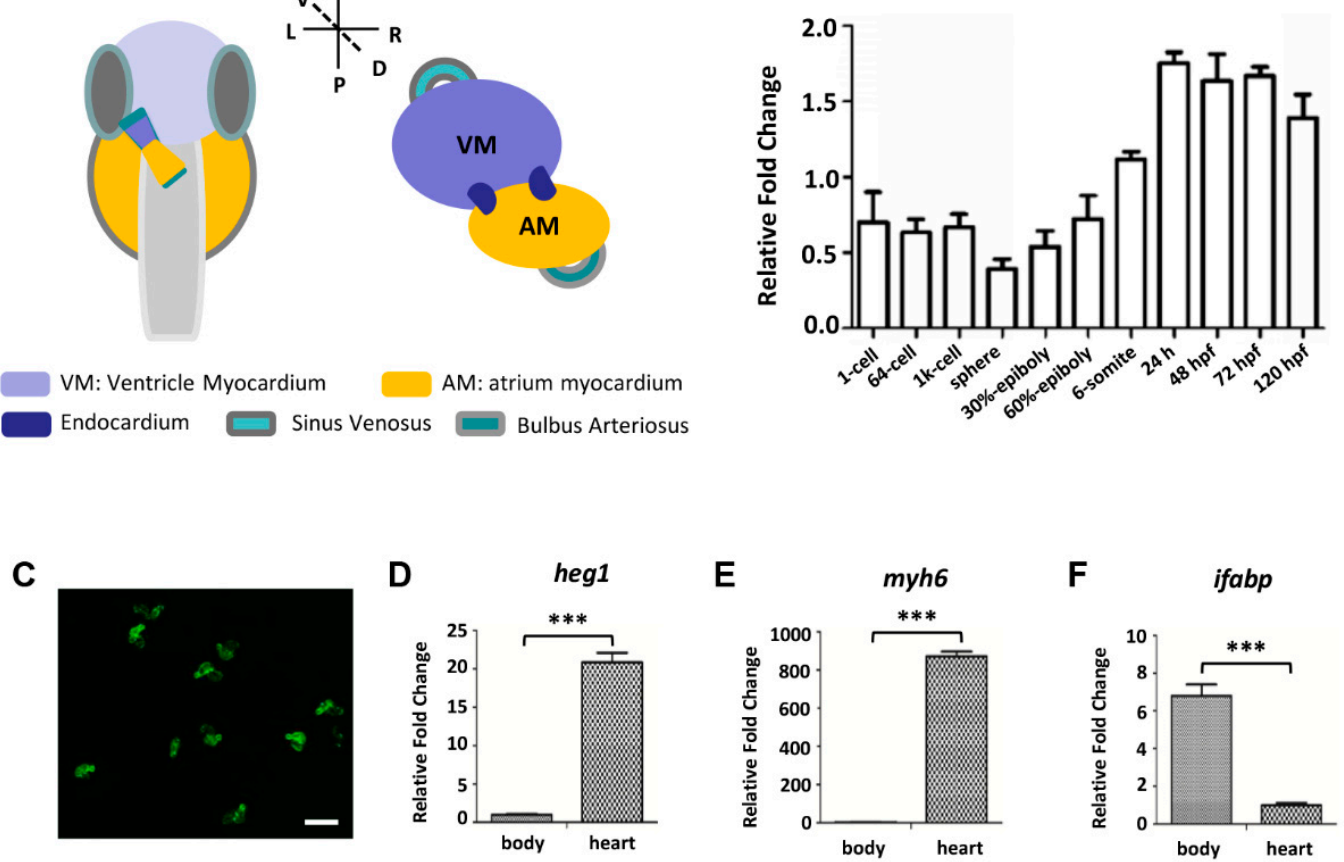

Figure 1. The expression level of heg1 in zebrafish heart tissue. (A) Overview of zebrafish embryos heart, dorsal view (VM: Ventricle Myocardium; AM: atrium myocardium). (B) qRT-PCR analysis of heg1 transcript levels from 1-cell to $120 \mathrm{hpf}$. (C) Purified heart tissues from $\mathrm{Tg}(\mathrm{cmlc} 2 \mathrm{eGFP})$ transgenic fish embryos at $72 \mathrm{hpf}$. heg1 (D) and myh6 (E) were highly expressed in purified heart tissue compared with the expression in body tissue as determined by qRT-PCR. (F) The expression level of ifabp in purified heart tissue was much lower than that in body tissue as analyzed by qRT-PCR. Scale bar: $100 \mu \mathrm{m} .{ }^{* * *}$ indicates $p<0.001$ by Student's $t$-test. 
Dilated cardiomyopathy (DCM) model and thrombosis model are two of the most popular zebrafish models that are widely used to mimic human CVD. For example, phenylhydrazine (PHZ) [11], photochemical excitation [12], and arachidonic acid (AA) [13] induced thrombosis models are used to evaluate antithrombotic drugs, and verapamil-induced heart failure model is used to assess the therapeutic effects of drug treatment. However, drug or physical injury modeling is a complex process, time-consuming, and characterized by phenotypic instability. Here, we hope to construct a stable zebrafish mutant which can be used for cardiovascular drug screening.

To date, there are more than 300 genes known to be related to congenital heart disease (CHD) [14]. The heart development protein with EGF-like domains 1 (HEG1) receptor is selectively expressed in endothelial cells and endocardium cells. During embryonic development, heg1, as an important intercellular adhesion cadherin protein, maintains the function and integrity of heart and blood vessels. In 2003, Mably et al. [15] first identified the heg1 gene through a zebrafish N-ethyl-N-nitrosourea (ENU) mutagenesis screening. Zebrafish heg1 mutants show cardiac valve defect, heart swelling, and delayed blood flow. In mice with $\mathrm{Heg} 1$ gene mutation, the vascular endothelial barrier is weakened, resulting in cardiovascular diseases such as sepsis, atherosclerosis, and multiple sclerosis [15-17].

Due to the advantages of the zebrafish system, it is gaining popularity, especially in studying the molecular mechanism of human diseases and related drug screening. Using genome editing technology, zebrafish strains can be created with specific pathogenic genes, which can mimic some of the disease gene mutations found in humans. The construction of zebrafish mutants and their application in drug screening have been reported. For example, Duchenne muscular dystrophy (DMD) is the most common and severe form of muscular dystrophy, which is caused by mutations in the Dystrophin (DMD) gene [18]. Zebrafish DMD mutants sapje and sapje-like exhibited dystrophic features of skeletal muscle at $72 \mathrm{hpf}$, which are ideal models for studying DMD [19]. Potential therapeutic drugs' screening carried in these DMD mutants identified at least seven chemicals that could rescue muscle phenotype found in the dystrophin-null fish [19]. Nav1.1 (SCN1A) mutation is one of the main causes of Dravet syndrome. Zebrafish scn1a mutants exhibited spontaneous abnormal electrographic activity, hyperactivity and convulsive behaviors, so they can simulate the clinical phenotype of Dravet syndrome patients. A large-scale drug screening based on 320 compounds identified that clemizole significantly inhibited convulsive behavior and electrographic seizures of mutant embryos, which confirmed the potential clinical significance of clemizole in the treatment of Dravet syndrome [20]. This represents that zebrafish mutants can be used to identify novel therapeutic drugs for a rapid and cost-effective purpose.

In this study, we generated the zebrafish heg1 mutants by CRISPR/Cas9 technology. Zebrafish heg1 mutant embryos developed pericardial swelling, pericardial edema, and decreased heart rate, which were similar to the pathophysiology characteristics observed in heart failure patients [14]. heg1 mutants' embryos also exhibited venous congestion, reduced cardiac output and blood flow velocity, which was consistent with the features of zebrafish thrombosis model. To assess the zebrafish heg1 mutant in cardiovascular drug screening, four Traditional Chinese Medicine (TCM) herbs (Salviae miltiorrhiza (Danshen), Radix astragali (Huangqi), Hirudo (Shuizhi) and Myrrha (Moyao)) and three Chinese herbal monomers (salvianolic acid B, paeoniflorin, ferulic acid) were used to treat heg1 mutant. Here, we provided a new zebrafish model of DCM and thrombosis, which could be applied for potential clinical CVD drug screening.

\section{Materials and Methods}

\subsection{Zebrafish Care and Maintenance}

All adult zebrafish were housed in aquaculture facility with a standard light cycle $(14 \mathrm{~h}$ light, $10 \mathrm{~h}$ dark) [21]. Embryos were obtained by natural crosses of wild-type (wt) or transgenic fish [22]. The following lines were used: $\mathrm{AB}$ strain, $\operatorname{Tg}(\mathrm{cmlc2}: e G F P)$ [23], and $\operatorname{Tg}(f l k 1: e G F P)$ [24]. All experimental procedures on zebrafish were approved by the Institutional Animal Care and Use 
Committee of Northwest University and carried out in accordance with the approved guidelines (NWU-AWC-20180601Z).

\subsection{Purification of Hearts from Zebrafish Embryos}

Hearts were extracted from $T g(c m l c 2:$ eGFP) embryos according to the method adapted from Burns [25]. A total of 200 to 300 embryos at $72 \mathrm{hpf}$ were placed into a $1.5 \mathrm{~mL}$ tube. Excess water was removed and $1 \mathrm{~mL}$ of L-15 medium containing $10 \%$ of fetal calf serum was added. A $5 \mathrm{~mL}$ syringe with a 20-gauge needle was used to destroy the pericardium and to release cardiac tissues by repeatedly passing embryos through syringe about 15 times. Fragmented embryos were placed in a $100 \mathrm{~mm}$ dish with cold L-15 medium; hearts were collected with a glass pipette under fluorescence microscope and placed in cold L-15 medium. After centrifugation at $4{ }^{\circ} \mathrm{C} 1000 \mathrm{~g}$ for $15 \mathrm{~min}$, the supernatant was discarded and the precipitated cardiac tissue was collected for further study.

\subsection{Generation of heg1 Mutant Zebrafish}

The procedure for CRISPR/Cas9 editing in zebrafish was described in detail in [26,27]. Zebrafish heg1 target sites were designed using the website https://www.crisprscan.org/?page= sequence [28] (2015-2019, Giraldez lab, New Haven, CT, USA). The provided sites were then screened in NCBI. heg1 guide RNA (gRNA) (5'- CCGTGTGCTTTTCACGGCCGC -3') that specifically targeted exon 1 in the zebrafish genome were chosen for the interruption of the heg1 gene. The gRNA was transcribed in vitro using the MAXIscript T7 kit (Ambion, Waltham, MA, USA). The mixtures including $100 \mathrm{pg}$ gRNA and 0.5pmol Cas9 protein (NEB, Ipswich, UK) were injected into one-cell stage embryos using PLI-100A Plus Pico Injector (Digitimer North America, Lauderdale, FL, USA). More than a dozen embryos were lysed and sequenced to examine gRNA efficiency, and the remaining embryos were raised to adulthood to obtain the mosaic founders. Mosaic zebrafish were crossed with AB wild-type zebrafish to obtain heg1 heterozygotes and genotyped by Sanger sequencing. heg $1^{+-}$were further crossed with the cardiovascular system specific transgenic reporter lines $\operatorname{Tg}(\mathrm{cmlc2}: E G F P)$ or $\operatorname{Tg}(f l k 1: E G F P)$ for phenotypic analysis. Genotyping primers of heg1 are summarized in Supplemental Table S1.

\subsection{Cardiac Phenotype Analysis}

Embryos were collected at 48, 72, and $96 \mathrm{hpf}$ for phenotype analysis. Zebrafish embryos were mounted in 5\% methylcellulose. Images were captured using a stereomicroscope (SMZ25, Nikon, Tokyo, Japan) for morphology observation. Photographs were quantified using NIS-Elements BR software (Ver5.30.00, Nikon, Tokyo, Japan) to determine the pericardial area and sinus venosus and bulbus arteriosus (SV-BA) distance per field of view.

\subsection{Blood Flow Rate and Heart Rate Statistics}

Video imaging of the red blood (RBCs) movement in the posterior cardinal vein (PVC) for $96 \mathrm{hpf}$ embryos was captured by stereomicroscope (SMZ25, Nikon, Tokyo, Japan). Using DanioScope software (DVOC-0041, Noldus, Wageningen Netherlands) to analyze the movement ratio of RBCs based on changes in pixel density, the result was expressed as a ratio of blood flow pixel value to surrounding tissue. The heart rate of embryos per unit of time was counted with a stopwatch and a counter, expressed as beats per minute (BPM).

\subsection{Real-Time Quantitative PCR ( $q R T-P C R)$ Analysis}

Total RNA was extracted from fresh zebrafish embryo tissues using TRIzol reagent (Ambion, Austin, TX, USA) as described in [29] with modifications. cDNA synthesis was performed using SuperScriptTM reverse transcriptase system (Invitrogen, Carlsbad, CA, USA). qRT-PCR was performed with SYBR Green PCR Master Mix (Kapa Biosystems, Boston, MA, USA) and ViiA 7 Real-Time PCR System (ThermoFisher, Waltham, MA, USA). Primer sequences are listed in Supplementary Table S1. 


\subsection{Whole-Mount in situ Hybridization and Red Blood Cell Staining Assay}

Digoxigenin (DIG)-labeled riboprobes of $c m l c 1$ were synthesized using RNA Labeling Kit (Roche, Basel, Switzerland). Whole-mount in situ hybridization (WISH) assays were performed as described previously $[29,30]$. Briefly, zebrafish embryos at different stages were collected and fixed in $4 \%$ paraformaldehyde at $4{ }^{\circ} \mathrm{C}$ overnight. After a 4-h pre-hybridization incubation at $65^{\circ} \mathrm{C}$, embryos were subsequently incubated with the antisense probes overnight. Anti-digoxigenin antibody (Roche, Basel, Switzerland) was used to bind the probes overnight at $4{ }^{\circ} \mathrm{C}$. The embryos were stained using $\mathrm{BM}$ purple (Roche, Basel, Switzerland), and photographed under Nikon SMZ25 microscope system.

The $4 \mathrm{dpf}$ embryos were fixed in $4 \%$ paraformaldehyde (PFA) for four hours at room temperature, then red blood cells (RBC) were stained by o-dianisidine solution comprised of $2.9 \mathrm{mM}$ 3,3'-Dimethoxybenzidine, $0.1 \mathrm{M} \mathrm{NaOAc}, 30 \% \mathrm{H}_{2} \mathrm{O}_{2}$, and 50\% absolute ethanol in the dark for $30 \mathrm{~min}$. Stained embryos were washed with PBST three times and stored in $80 \%$ glycerol for imaging using Nikon SMZ25 Microscope.

\subsection{Drug Preparation}

The TCM herbs (Salviae miltiorrhiza (Danshen), Radix astragali (Huangqi), Hirudo (Shuizhi) and Myrrha (Moyao)) were provided from Buchang Pharmaceuticals (Shaanxi, China). The Salviae miltiorrhiza, Radix astragali and Hirudo were macerated in $50 \mathrm{~mL}$ distilled water for $30 \mathrm{~min}$, and were ultrasonicated $(250 \mathrm{~W}, 50 \mathrm{kHz})$ for $30 \mathrm{~min}$, evaporated to dry powder and dissolved in $\mathrm{H}_{2} \mathrm{O}$ to a final concentration $1 \mathrm{~g} / \mathrm{mL}$. Myrrha was prepared by alcohol extraction and dissolved in DMSO. The stock concentrations of four herbs were $1 \mathrm{~g} / \mathrm{mL}$.

Salvianolic acid B (SS8100), paeoniflorin (SP8030), ferulic Acid (SF8030) and aspirin (IA0550) were purchased from Solarbio Company (Beijing, China). All monomers were dissolved in DMSO at stock concentration of $10 \mathrm{mM}$.

\subsection{Assessment Effects of Drugs on heg1 Mutant}

heg1 mutants embryos at $48 \mathrm{hpf}$ were collected and placed into 6-well plates. To optimize the concentrations of TCM herbs and monomers, the following doses were used: Radix astragali (1100, 1300, 1600, 2000, and $2200 \mathrm{mg} / \mathrm{L})$, Salviae miltiorrhizae (1800, 2000, 2200, 3000, and $3800 \mathrm{mg} / \mathrm{L})$, Hirudo (300, 400, 500, 1000, and $2000 \mathrm{mg} / \mathrm{L})$, Myrrha (50, 100, 150, 300, and $400 \mathrm{mg} / \mathrm{L})$, salvianolic acid B (5, 10, 15, 20, $30 \mu \mathrm{M})$, paeoniflorin $(5,10,15,20,30 \mu \mathrm{M})$, ferulic acid $(5,10,15,20,30 \mu \mathrm{M})$, and aspirin $(60,80,100$, $120,140 \mu \mathrm{M}$ ) for 2 days, respectively. The recovery rate of venous thrombosis was recorded at the end of the treatment; 40 embryos per group were used for statistics analysis.

The optimal concentrations of Radix astragali $1300 \mathrm{mg} / \mathrm{L}$, Salviae miltiorrhiza $2000 \mathrm{mg} / \mathrm{L}$, Hirudo $400 \mathrm{mg} / \mathrm{L}$, and Myrrha $100 \mathrm{mg} / \mathrm{L}$, salvianolic acid B $20 \mu \mathrm{M}$, paeoniflorin $15 \mu \mathrm{M}$, ferulic acid $5 \mu \mathrm{M}$ and aspirin $120 \mu \mathrm{M}$ were treated on heg1 mutant embryos at $48 \mathrm{hpf}$ for 2 days, respectively. Control groups were treated with $0.1 \%$ DMSO or $0.9 \%$ sodium chloride to confirm that the solvent did not have an adverse effect on embryos. After treatment, ten embryos from each group were randomly selected for phenotype observation, cardiac morphology measurement, blood flow rate and heartbeat statistics' analysis.

\subsection{Statistical Analysis}

All experiments were repeated at least three times. Data analysis was performed by using SPSS Statistical Package version 17 (IBM, Armonk, NY, USA). Student's $t$-test was applied for comparisons among different groups. All data are presented as mean $\pm \mathrm{SE}$, and $p<0.05$ was considered significant statistically. 


\section{Results}

\subsection{Zebrafish heg1 was Highly Expressed in Cardiac Tissue}

Levels of heg1 transcripts were analyzed by qRT-PCR. As shown in Figure 1B, the maternal mRNA signals of heg1 could be detected during the cleavage (1-cell, 64-cell). The zygotic heg1 was expressed through the blastula stage (1k-cell, sphere), gastrulation (30\%-epiboly, 60\%-epiboly), somitogenesis (6-somite), reached the highest level at $24 \mathrm{hpf}$, and maintained high expression until $120 \mathrm{hpf}$, as detected (Figure 1B). To further investigate the tissue-specific expression of heg1, zebrafish embryonic hearts were dissected and collected at $72 \mathrm{hpf}$ (Figure 1C). The expression of heg1 in heart was twenty times higher than that in body tissues (Figure 1D). As tissue-specific markers, myosin heavy chain 6 (myh6) is highly expressed in atrium [31], and fatty acid binding protein 2 $(f a b p 2 / i f a b p)$ is known to be specifically expressed in intestinal epithelium [32]. As shown in Figure 1E,F, the expression of myh6 in zebrafish cardiac tissue was more than 800 times higher than that in other tissues, while the expression level of ifabp in purified cardiac tissues was very low (Figure 1E,F). The results of quantitative analysis of gene expressions revealed that heg1 gene was much more abundant in heart tissue than in other tissues, which strongly indicated that heg1 played an important role in cardiac development.

\section{2. heg1 Deficient Zebrafish Was Generated Using CRISPR/Cas9 Technology}

Zebrafish heg1 gene is located on chromosome 9 and consists of 13 exons and 12 introns (Figure 2A). heg1 gRNA comprising a 21-base sequence was designed for the gene-specific (Figure 2A). We were able to generate a 25-nt deletion in exon1, which led to a frameshift and a premature stop codon (TGA), resulting in a truncated protein with only 12 amino acids left (Figure 2A,B). The mutation disrupted all known functional domains of heg1 protein. qRT-PCR analysis editing of exon 1 showed that the transcript of heg1 mRNA was significantly eliminated in heg $1^{\Delta 25}$ embryos (Figure 2C), which might be due to the effect of nonsense-mediated decay (NMD) [32]. Thus, it indicated that heg1 $1^{\Delta 25}$ was a loss-of-function mutation.

\subsection{Abnormal Cardiac Formation in heg $1^{\Delta 25}$ Mutants}

Obvious cardiovascular malformation of heg $1^{\Delta 25}$ mutants were observed from $48 \mathrm{hpf}$. The mutants exhibited progressively severe edema, first in the pericardial sac and then around the eyes and yolk sac (Figure 3A, $\mathrm{A}^{\prime}$ ). The heart wall of the heg $1^{\Delta 25}$ mutant is grossly distended, and congestion was seen both in the heart and in the posterior cardinal vein (Figure 3A, $\mathrm{A}^{\prime}$ ). Cmlc (cardiac myosin light chain) family specifically encodes myosin and is expressed in the heart during the development of the cardiovascular system [33]. The WISH of cmlc1 (Figure 3B, $\mathrm{B}^{\prime}$ ) and bmp4 (Figure S1B,B') showed enlarged heart size in $h e g 1^{\Delta 25}$ mutant with overtly expanded atrium. To better analyze the cardiac defects in heg1 $1^{\Delta 25}$ mutant, heg $1^{\Delta 25}$ was crossed with the cardiomyocyte specific transgenic reporter line $\mathrm{Tg}(\mathrm{cmlc2}: e G F P)$ to produce the bi-genic line $\left(h e g 1^{\Delta 25} ; \mathrm{cmlc2}: e G F P\right)$. This $\left(h e g 1^{\Delta 25} ; \mathrm{cmlc} 2: e G F P\right)$ line also showed enlargement of heart chambers (Figure $3 \mathrm{C}, \mathrm{C}^{\prime}$ ).

Under normal circumstances, the heart is beating spontaneously and regularly. The change in heart rhythm or interference may be the cause of cardiac malfunction. In heg $1^{\Delta 25}$ mutant embryos, heart rate was markedly reduced compared to wt embryos (Figure 3D). Meanwhile, the cardiac area in the heg $1^{\Delta 25}$ mutant was almost double that of the wt embryos (Figure 3E), reflecting severe heart edema and pericardial effusion. The distance between sinus venosus and bulbus arteriosus (SV-BA) is an indicator to measure the degree of cardiac looping. heg $1^{\Delta 25}$ mutant exhibited a significant prolongation of the SV-BA distance (Figure $3 F$ ). The zebrafish heg $1^{\Delta 25}$ mutant cardiac defects was resembled to human heart failure (HF) caused by dilated cardiomyopathy (DCM). 
A

heg1

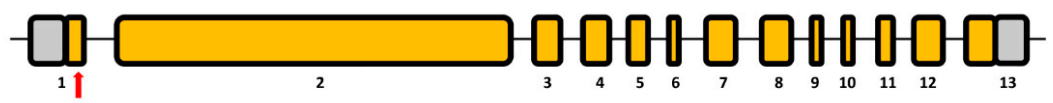

taatacgactcactataCCGTGTGCTTTTCACGGCCGC gttttagagcta gaaatagc

Target site

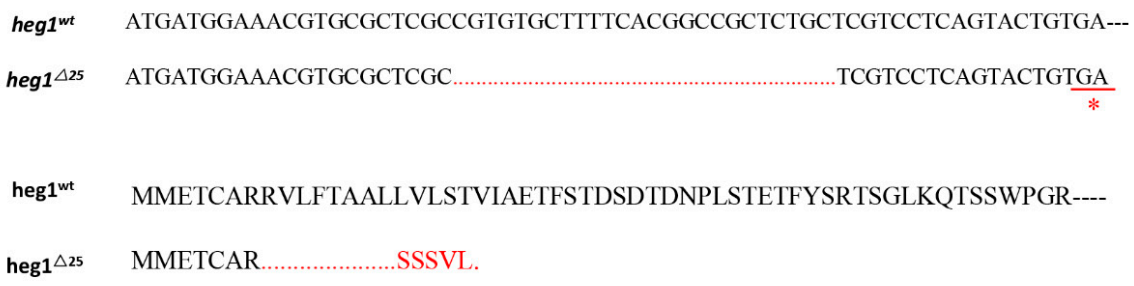

heg1 $\triangle 25$ MMETCAR .....................SSSVL,

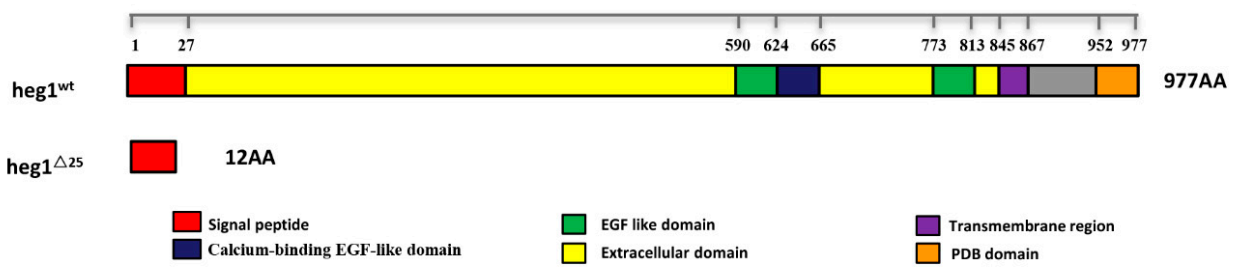

B

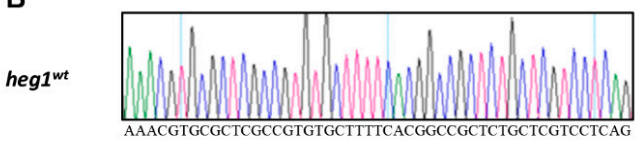

$\operatorname{heg} 1 \triangle 25$

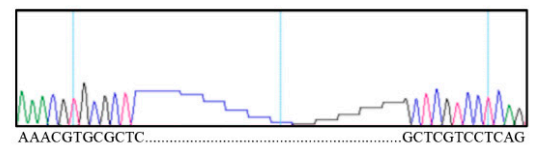

C

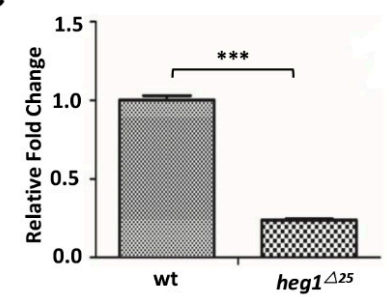

Figure 2. The generation of heg1 deficient zebrafish using CRISPR/Cas9 technology. (A) Schematic view of zebrafish heg1 genomic and protein domains. The gRNA sequence for the exon 1 targeting site was labelled by a red line. The CRISPR/Cas9-induced mutation (25-base deletion) in heg1 exon1 and a truncated 12 amino acids at the N-terminus were shown. (B) Sanger sequencing confirmed the 25-nt deletion mutation. (C) qRT-PCR confirmation that heg1 expression was significantly decreased in heg $1^{\Delta 25}$ embryos, $n=30$ embryos per group. Data are represented as mean \pm SE. ${ }^{* *} p<0.001$.

\subsection{Abnormal Vascular Development and Blood Stagnation in heg $1^{\Delta 25}$ Mutants}

Cardiac malformations are usually accompanied by vascular dysplasia and poor blood circulation. Embryonic endothelial cells can be easily observed in zebrafish using transgenic fluorescent zebrafish $T g(f l k 1: e G F P)$ line. heg1 ${ }^{\Delta 25}$ was crossed with $T g(f l k 1: e G F P)$ to produce the bi-genic line $\left(h e g 1^{\Delta 25} ; f l k 1: e G F P\right)$ for vascular malformation observation. In heg $1^{\Delta 25}$ mutant embryos, blood often accumulated and coagulated in heart chambers or blood vessels (Figure 3A, $\mathrm{A}^{\prime}$ and Figure $4 \mathrm{~A}, \mathrm{~A}^{\prime}$ ). Fluorescence microscope images further revealed the dilation of dorsal aorta (DA) lumen in heg $1^{\Delta 25}$ embryos at $96 \mathrm{hpf}$ (Figure 4A,A'). Red blood cells (RBCs) staining in heg $1^{\Delta 25}$ embryos at $96 \mathrm{hpf}$ showed the accumulation of RBCs not only in the heart, but also in the posterior cardinal vein (PCV) and tail vein (TV) (Figure $4 B, B^{\prime}, C, C^{\prime}$ ). The movement of the RBCs in the tail vein of $96 \mathrm{hpf}$ embryos was validated by using DanioScope software. It could be clearly seen that the relative movement of RBCs in heg $1^{\Delta 25}$ embryos was almost abolished (Figure 4D); qRT-PCR results also confirmed that thrombotic markers, such as $f 2$ and tbxasl, were also abnormally increased in heg $1^{\Delta 25}$ mutants (Figure S1A). 

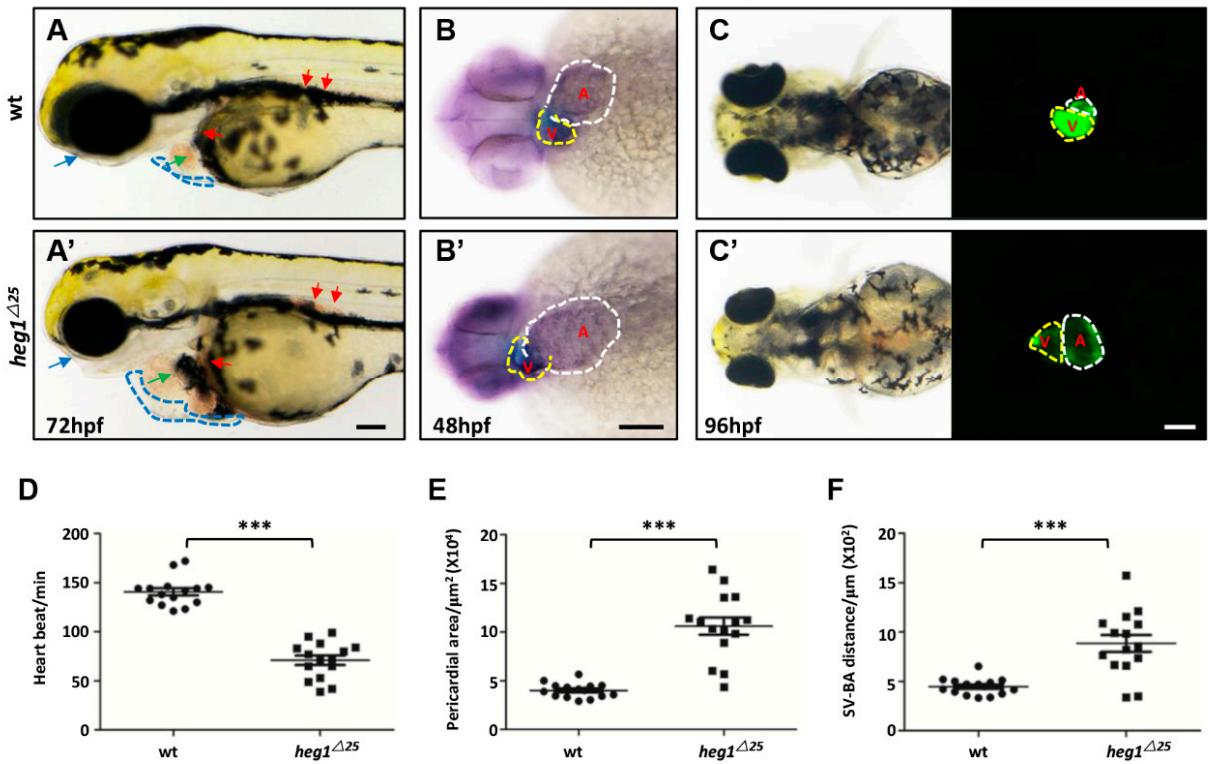

\section{E}
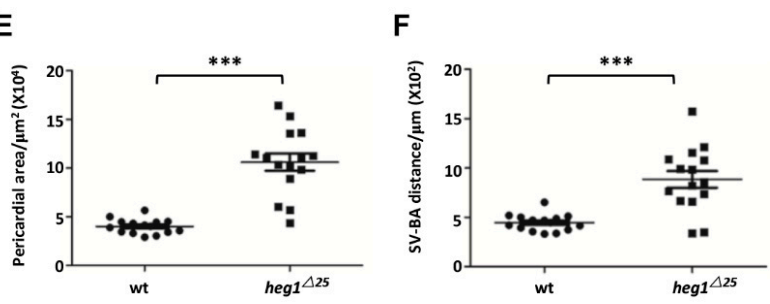

Figure 3. heg1 deficiency leaded to abnormal cardiac development in zebrafish embryos. (A, $\left.\mathbf{A}^{\prime}\right)$ Lateral view of zebrafish larvae at $72 \mathrm{hpf}$. Representative images of the heg $1^{\Delta 25}$ and wt embryos, exhibiting pericardial edema (blue dotted-line boxes), enlarged heart size (green arrow), venous congestion (red arrow), and eye edema (blue arrow). (B,, $\left.\mathbf{B}^{\prime}\right)$ Representative images of the heg $1^{\Delta 25}$ and wt embryos at $48 \mathrm{hpf}$ stained for the heart marker cmlc1. Note the enlargement heart in heg $1^{\Delta 25}$ mutants (V: Ventricular, yellow dotted-line boxes; A: atria, white dotted-line boxes, ventral view). $\left(\mathbf{C}, \mathbf{C}^{\prime}\right)$ The heart morphology was delineated by $\mathrm{Tg}(\mathrm{cmlc2}$ :GFP) (ventral view). (D) Heart rate in wt and heg $1^{\Delta 25}$ mutant zebrafish larvae $\left(n=15\right.$ embryos/group). (E) The pericardial area in wt and heg $1^{\Delta 25}$ mutant zebrafish larvae ( $n=15$ embryos/group). (F) The SV-BA distance in wt and heg1 ${ }^{\Delta 25}$ mutant zebrafish larvae ( $n=15$ embryos /group). Scale bar: $100 \mu \mathrm{m}$. ${ }^{* * *}$ indicates $p<0.001$ by Student's $t$-test.

Furthermore, the expressions of cardiovascular markers were significantly changed as detected by qRT-PCR analysis in heg $1^{\Delta 25}$ mutant (Figure 3E). As shown in Figure 4E, the expressions of $c m l c 2$ and myh6, specific markers for heart tissue, were abnormally increased in heg $1^{\Delta 25}$ mutant embryos compared with wt embryos. Besides, the expressions of vascular markers, such as sox $7, f l k 1, f l t 4$, $c-m y b$ [34] were also abnormally increased in heg $1^{\Delta 25}$ mutant embryos (Figure $4 \mathrm{E}$ ). This indicated cardiovascular malformations by loss of heg1 function in zebrafish.

In general, blood flow velocity of heg $1^{\Delta 25}$ mutants was significantly lower than that of wt embryos. The venous congestion of heg $1^{\Delta 25}$ mutants was very similar to that of zebrafish thrombosis model induced by physical and/or chemical treatments.

\subsection{Application of heg $1^{\Delta 25}$ Mutants in Screening TCM Herbs for Cardiovascular Diseases Treatment}

In order to verify the feasibility of heg $1^{\Delta 25}$ mutants as a model of human heart failure and thrombosis, four known TCM herbs for the treatment of cardiovascular diseases or as a component of compound Chinese medicines for the treatment of cardiovascular diseases were selected to verify the model. Radix astragali (Huangqi) has been implicated in promoting endothelial cell proliferation and migration during angiogenesis [35,36]. The active ingredients of Salviae miltiorrhiza (Danshen) have antithrombotic and anticoagulant effects, and promote vascular remodeling and angiogenesis [37]. Hirudo (Shuizhi) can inhibit vascular smooth muscle cell (VSMC) proliferation, and the VSMC proliferation plays an important role in the formation of atherosclerotic plaque [38]. Myrrh (Moyao) is usually used as a component of compound Chinese medicines for the treatment of cardiovascular diseases, applied as an anticoagulant [39]. 

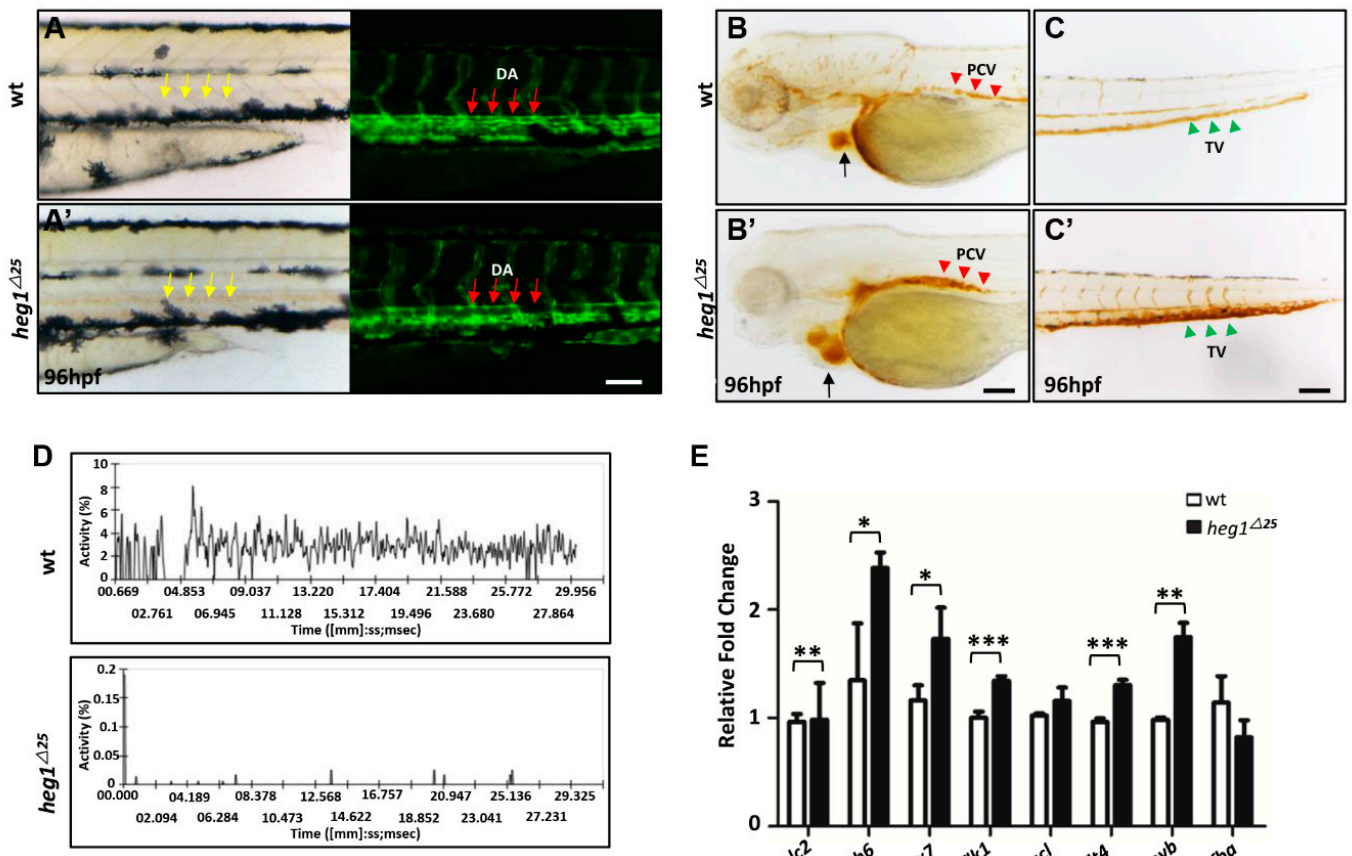

E

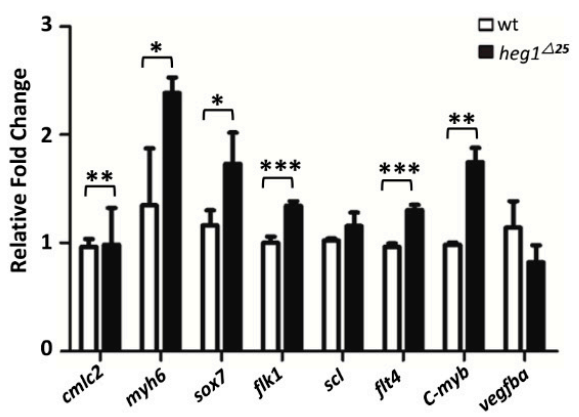

Figure 4. heg1 deficiency leaded to poor blood flow and abnormal vascular development in zebrafish embryos. (A) Lateral view of zebrafish larvae at $96 \mathrm{hpf}$. Representative images of the heg ${ }^{\Delta 25}$ and wt embryos, exhibiting blood congestion (yellow arrows), and dilation of dorsal aorta (DA) lumen (red arrows) in caudal vein. (B,C) Representative images of the heg $1^{\Delta 25}$ and wt embryos, showing red blood cells (RBCs) accumulation in posterior cardinal vein (PCV) (red arrowhead), tail vein (TV) (green arrowhead) and heart (black arrow). (D) The movement ratio of RBCs based on changes in pixel density of PVC. (E) The expressions of cardiovascular markers, as determined by qRT-PCR, were significantly changed in heg $1^{\Delta 25}$ mutants at $48 \mathrm{hpf}$. Data are represented as mean $\pm \mathrm{SE}$ from three independent experiments, ${ }^{*} p<0.05,{ }^{* *} p<0.01$, and ${ }^{* * *} p<0.001$ (Student's $t$-test).

Zebrafish heg1 $1^{\Delta 25}$ embryos at $48 \mathrm{hpf}$ were treated with each drug to determine the optimal concentration for recovery. After two days of treatment, heg $1^{\Delta 25}$ embryos treated with Radix astragali or Salviae miltiorrhiza exhibited significantly reduced venous congestion in PCV (red arrow) (Figure 5A), and the relative movement of RBCs in PCV were also obviously recovered (Figure 5A). Hirudo also showed a certain effect on venous thrombosis (red arrow), but had no obvious effect on the recovery of RBCs' movements (Figure 5A). The optimal concentrations of the four drugs are shown in Figure 5B: Radix astragali $1300 \mathrm{mg} / \mathrm{L}$, Salviae miltiorrhiza $2000 \mathrm{mg} / \mathrm{L}$, Hirudo $400 \mathrm{mg} / \mathrm{L}$, and Myrrha $100 \mathrm{mg} / \mathrm{L}$, respectively. We next further analyzed the restoring phenotype of heg $1^{\Delta 25}$ mutants at the optimal concentration of each drug. The heart rate of $h e g 1^{\Delta 25}$ at $96 \mathrm{hpf}$ was reduced to $80 \pm 2.6 \mathrm{BPM}$ compared with the heart rate of $130 \pm 5.5$ in wt group. Treatment with Radix astragali results in mutants' heart rate returning to $121 \pm 2$ BPM. Salviae miltiorrhiza and Hirudo also showed different degrees of recovery to heart rate (Figure $5 \mathrm{C}$ ). Since heg $1^{\Delta 25}$ embryos had an enlarged atrium and ventricle, the SV-BA distance and the pericardial area were increased aberrantly in heg $1^{\Delta 25}$ embryos. Radix astragali and Salviae miltiorrhiza treatment could reduce the abnormal SV-BA distance and significantly restore the pericardial edema of heg1 $1^{\Delta 25}$ embryos (Figure 5A,D,E). Therefore, the cardiovascular phenotype of heg $1^{\Delta 25}$ mutants was restored in different degrees under the treatment of Radix astragali, Salviae miltiorrhiza, and Hirudo.

\subsection{Application of heg1 $1^{\Delta 25}$ mutants in Screening monomers for Cardiovascular Diseases Treatment}

To further validate the reliability and diagnostic significance of the heg $1^{\Delta 25}$ mutants in drug screening, we selected three bioactive components of TCM for the treatment of cardiovascular diseases to verify their rescue effect. Salvianolic acid B can promote angiogenesis and lesion plaque stability, 
and reduce acute myocardial infarction and atherosclerosis [40]. Paeoniflorin can inhibit acute myocardial infarction, ischemic stroke and atherosclerosis, and has a function of reducing acute myocardial infarction, ischemic stroke and atherosclerosis [41,42]. Ferulic Acid exhibits hypoglycemic and anti-oxidant effects, and has a certain therapeutic effect on diabetic cardiomyopathy (DCM) in rats [43,44]. Aspirin (acetylsalicylic acid, ASP) is one of the main therapeutic interventions for almost all patients with increased risk of thrombosis, and here it serves as a positive control.

As shown in Figure 6, salvianolic acid B exhibited the best recovery ability to heg $1^{\Delta 25}$ mutants in venous thrombosis (Figure 6A, red arrow), RBCs movements (Figure 6A), heart rate (Figure 6C), SV-BA distance (Figure 6D) and the pericardial area (Figure 6E) at the optimal concentration (Figure 6B), which was similar to that of aspirin. However, paeoniflorin and ferulic acid had no significant effect on the recovery of heg $1^{\Delta 25}$ mutant cardiovascular phenotype.
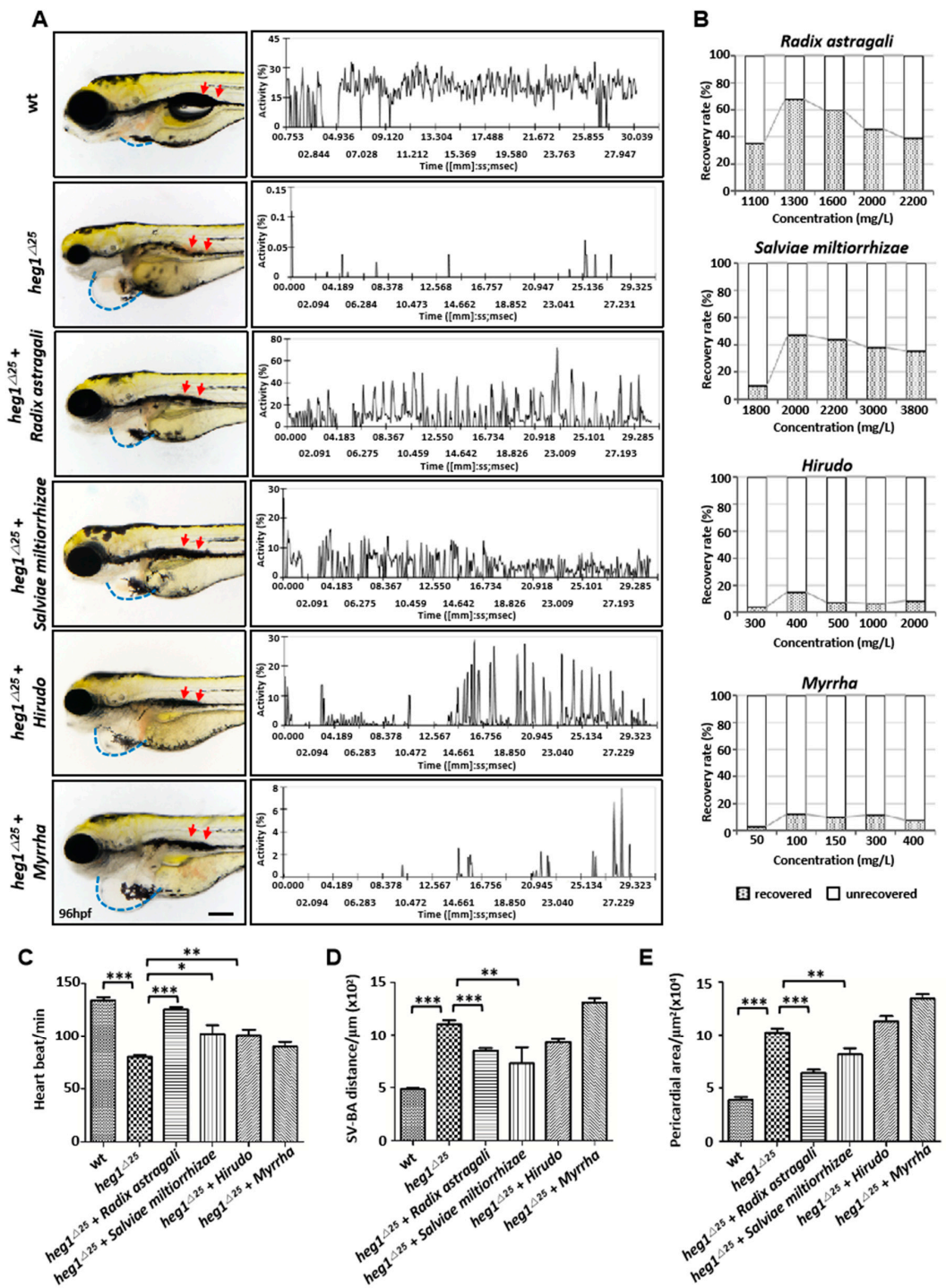

Figure 5. TCM pharmacological validation of $h e g 1^{\Delta 25}$ zebrafish mutant. (A) Phenotype observation and the relative movement of RBCs in PVC analysis of heg1 $1^{\Delta 25}$ embryos treated with four TCM herbs. (B) The optimal concentrations of the four drugs, as indicated as Radix astragali $1300 \mathrm{mg} / \mathrm{L}$, Salviae miltiorrhiza $2000 \mathrm{mg} / \mathrm{L}$, Hirudo $400 \mathrm{mg} / \mathrm{L}$, and Myrrha $100 \mathrm{mg} / \mathrm{L}$, respectively. $\mathrm{N}=40$ embryos for each concentrations. (C) Comparison of heart rate of zebrafish embryos in each group. (D) Comparison of SV-BA distance of zebrafish embryos in each group. (E) Comparison of pericardial area of zebrafish embryos in each group. Data are represented as mean \pm SE from three independent experiments, ${ }^{*} p<0.05,{ }^{* *} p<0.01$, and ${ }^{* * *} p<0.001$ (Student's $t$-test). 
A

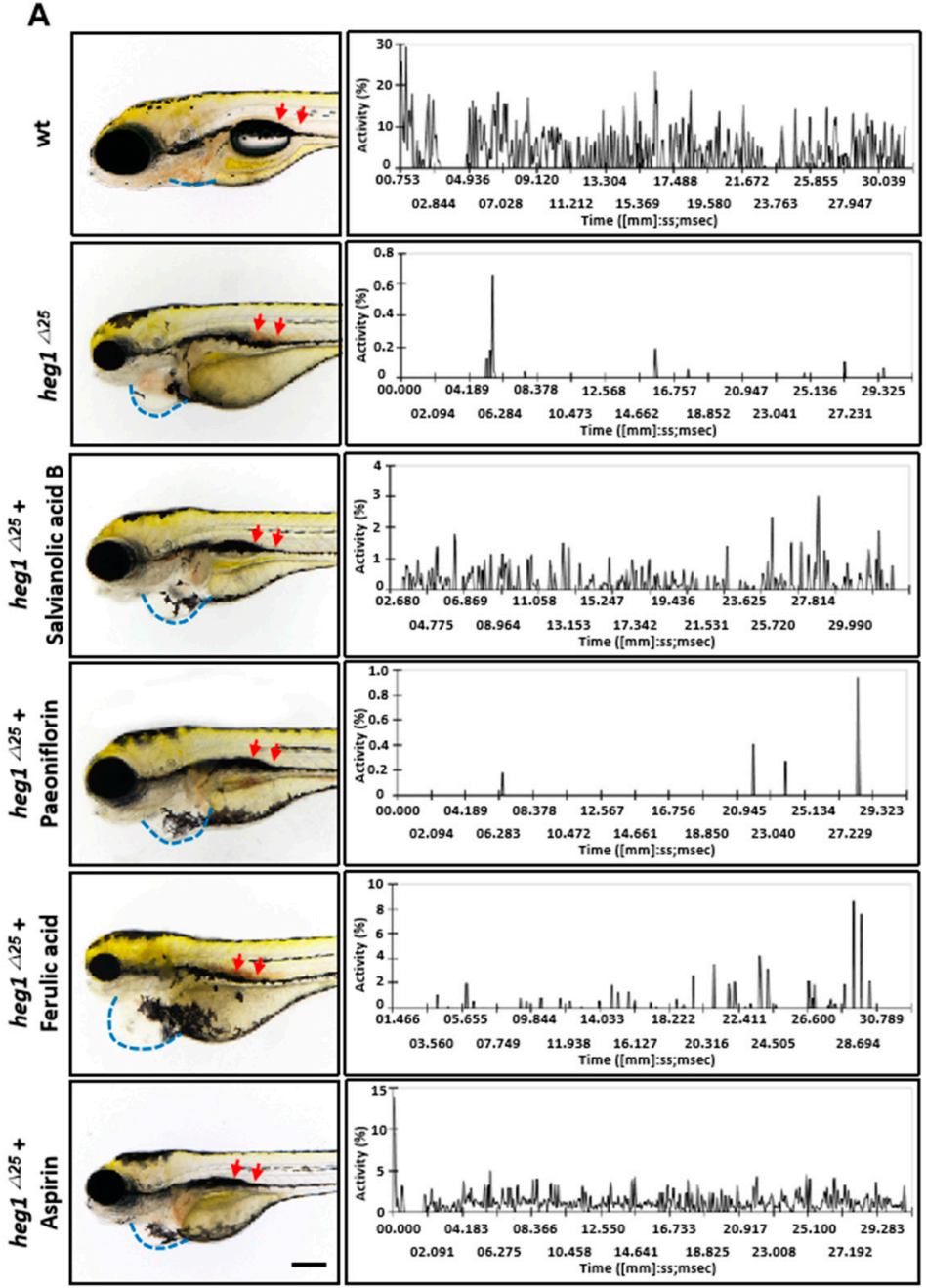

B
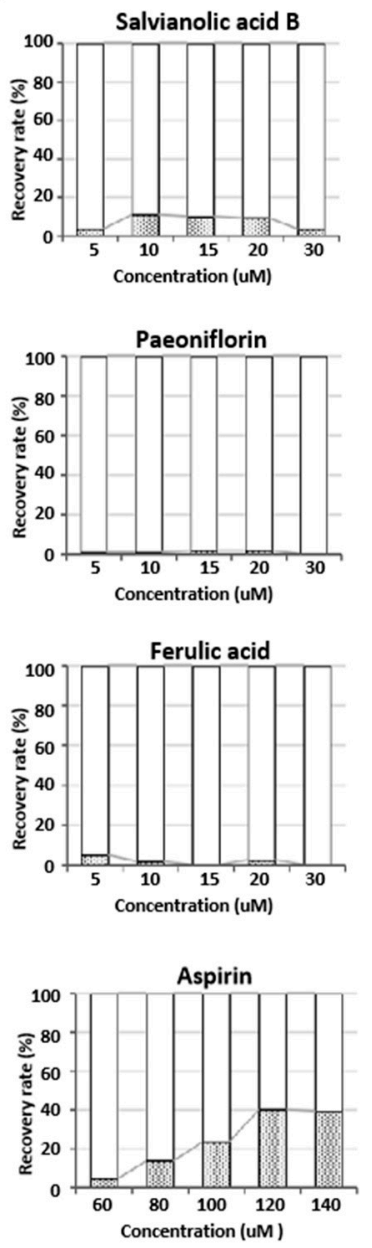

圆 recovered $\square$ unrecovered c

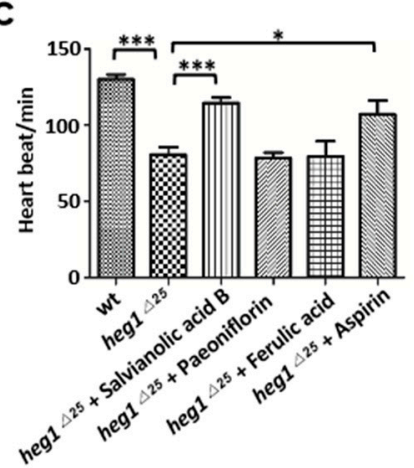

D

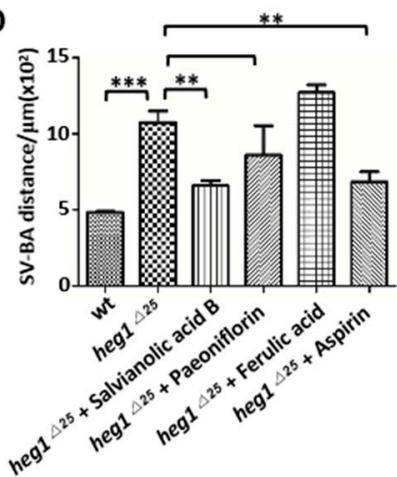

E

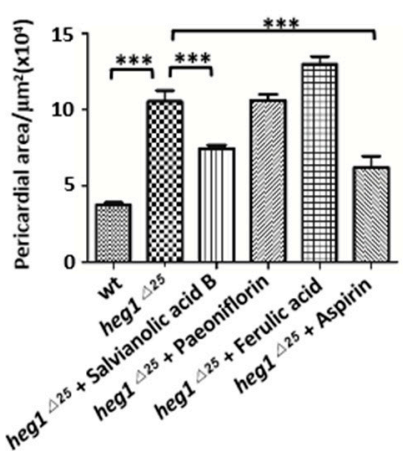

Figure 6. Monomers pharmacological validation of heg1 $1^{\Delta 25}$ zebrafish mutants. (A) Phenotype observation and the relative movement of RBCs in PVC analysis of $h e g 1^{\Delta 25}$ embryos treated with four monomers. (B) The optimal concentrations of the four monomers, salvianolic acid B, paeoniflorin, ferulic acid and aspirin. $\mathrm{N}=40$ embryos for each concentration. (C) Comparison of heart rate of zebrafish embryos in each group. (D) Comparison of SV-BA distance of zebrafish embryos in each group. (E) Comparison of pericardial area of zebrafish embryos in each group. Data are represented as mean \pm SE from three independent experiments, ${ }^{*} p<0.05,{ }^{* *} p<0.01$, and ${ }^{* * *} p<0.001$ (Student's $t$-test).

\section{Discussion}

The heg $1^{\Delta 25}$ zebrafish mutant described here is a typical model that recapitulates features of human cardiovascular diseases. We had shown that heg1 $1^{\Delta 25}$ mutants exhibited DCM and venous 
thrombosis, including cardiac dilation, lower heart rate, PCV congestion, and reduced blood circulation. Additional molecular analysis of heg1 $1^{\Delta 25}$ mutants showed that the expression levels of cardiovascular-development-related genes were abnormal. Further detailed analysis revealed that $h e g 1^{+/ \Delta 25}$ heterozygous embryos have no abnormal phenotype in comparison with wt embryos (Figure S2). In terms of the drug screening application. Furthermore, four TCM herbs (Salviae miltiorrhiza, Radix astragali, Hirudo and Myrrha) and three Chinese herbal monomers (salvianolic acid B, paeoniflorin, and ferulic acid) were used to treat heg $1^{\Delta 25}$ mutants. We found that Salviae miltiorrhiza, Radix astragali, and salvianolic acid B could significantly restore the formation of thrombosis and relieve the heart failure in dilated cardiomyopathy of $h e g 1^{\Delta 25}$ mutant. Our studies indicated that the $h e g 1^{\Delta 25}$ mutant could be a valuable tool for the understanding and treatment of CVD.

In terms of those currently known, the therapeutic effects of TCM on CVDs are reflected by attenuating the damage of cardiomyocytes, endothelial cells (ECs), vascular smooth muscle cells (VSMCs) and macrophages/monocytes (M\&Ms) [45]. The four TCM herbs and three monomers used in our study are commonly used medications for the treatment or prevention of CVD [45-47], while they have different targets and effects in vivo. Among the three monomers, salvianolic acid B exhibited the best dose-dependent recovery effect on $h e g 1^{\Delta 25}$ mutant. Previous studies revealed that salvianolic acid B could inhibit the expression of vascular EGF protein in HUVEC cells by modulation of the ERK signaling pathway, as a result, to attenuate the disorganization of vascular endothelial-cadherin [47]. Salvianolic acid B also could inhibit TNF $\alpha$-induced endothelial permeability [48]. Combined with the characteristics of the heg1 ${ }^{\Delta 25}$ mutant, the enhanced adhesion of endothelial cells might be the reason for phenotype recovery in the treatment group. Salvianolic acid B is the extract of Salviae miltiorrhiza. Compared with salvianolic acid B, Salviae miltiorrhiza treatment group had a better curative effect. Salviae miltiorrhiza is widely used in the treatment of angina pectoris and acute ischemic stroke. Its effects mainly include dilation of coronary artery, improvement in microcirculation, inhibition of platelet adhesion and aggregation. Lin et al. reported that Salviae miltiorrhiza could reduce the phosphorylation of myosin light chain and facilitate the contraction of smooth muscle cells [45]. Salviae miltiorrhiza contains two types of major bioactive components, including water-soluble salvianolic acid and fat-soluble tanshinone. Rat acute myocardial infarction (MI) model experiment showed that salvianolic acid and tanshinone both could delay the development of ischemia by decreasing the infarct size and improving the systolic function after myocardial infarction [49]. Tanshinone acts in inhibition of intracellular calcium and cell adhesion. Salvianolic acid can mediate in oxidative stress genes downregulation, G-protein-coupled receptor activities and cell apoptosis in the late stage of ischemia [50]. The effect of various active components may be the reason why Salviae miltiorrhiza is more effective than salvianolic acid B.

In addition to Salvia miltiorrhiza and salvianolic acid B, Radix astragali also has a good effect on the treatment of heg $1^{\Delta 25}$ mutant mutant thrombosis and heart failure in a dose-dependent manner. However, some monomers such as paeoniflorin, and ferulic acid had no significant dose-dependent effect compared with aspirin. As seen in our results, TCM herbs seems to have a better therapeutic effect than herbal monomers. This may be related to the characteristics of the "multi-component, multi-target, and multi-pathway" of TCM [51]. To date, many TCM have not been fully studied, and their mechanism in the prevention and treatment of cardiovascular diseases are still unclear [52]. Thus, our study further supports that TCM are valuable in the prevention and treatment of CVD.

The zebrafish system is becoming more and more popular in studying the molecular mechanism of human diseases and drug screening. However, as a lower vertebrate model system, zebrafish heg $1^{\Delta 25}$ mutant is inevitably limited as a DCM or venous thrombosis model. Zebrafish heart has only one atrium and one ventricle; the anatomical structure of the cardiovascular system is much different from that of humans [4], so heg $1^{\Delta 25}$ mutants cannot accurately reflect the phenotype of human cardiovascular diseases. Further work is needed to verify the efficacy and safety of the screened drugs in other vertebrate model systems, such as mice. Different from the single phenotype obtained by drug modeling (such as terfenadine-induced dilated cardiomyopathy model [53]), the heg ${ }^{\Delta 25}$ mutant, 
as a congenital gene mutation model, has multiple developmental defects, which may bring some difficulties to specific drug screening and drug efficacy evaluation. However, as a gene knockout model, heg $1^{\Delta 25}$ mutant maybe more sensitive to heg1-related signaling pathways and regulatory genes, rather than being used in general drug screening for a broad range of pathologies $[19,20]$. These aspects must be taken into consideration in future studies and applications of heg $1^{\Delta 25}$ mutant. This will help us to further understand the regulatory pathways that drive these phenotypes, and even may even help us to screen relevant gene-targeted drugs.

Overall, zebrafish is an ideal model for screening and optimizing the timing and dosage of drugs because it can easily produce many embryos and allow the rapid evaluation of drug therapy effects. As a model of cardiovascular disease, heg $1^{\Delta 25}$ mutant zebrafish has important advantages in the pharmacological research of CVD in the future, and will also contribute to the in-depth study on the pathogenesis of CVD.

\section{Conclusions}

CVD is one of the most frequent reasons for morbidity and mortality in the world. The heg $1^{\Delta 25}$ zebrafish mutant we described here can be used as an effective model for the study of heart failure and venous thrombosis caused by human DCM. Some TCM herbs such as Salviae miltiorrhiza, Radix astragali, and monomers such as salvianolic acid $\mathrm{B}$, have promising therapeutic effects on the heart failure and venous thrombosis of heg $1^{\Delta 25}$ mutants. Taken together, the heg $1^{\Delta 25}$ mutant strain has broad application prospects in drug screening and therapeutic treatment of CVD, and should be widely used in future.

Supplementary Materials: The following are available online at http://www.mdpi.com/2218-273X/10/11/1542/s1, Table S1: List of all primer sequences. Figure S1: Analysis of heg $1^{\Delta 25}$ mutant embryos. Figure S2: Comparison of $\mathrm{wt}, h e g 1^{+/ \Delta 25}$ heterozygous and heg1 $1^{\Delta 25}$ homozygous mutants.

Author Contributions: Conceptualization, J.T.; methodology, S.L. and M.H.; software, H.L.; validation, Z.W., Y.K. and Z.L.; formal analysis, S.L.; investigation, Z.W.; resources, Z.L.; data curation, M.H.; writing-original draft preparation, S.L.; writing-review and editing, J.T. and S.L.; visualization, S.L.; supervision, J.T.; project administration, Y.K.; funding acquisition, J.T. All authors have read and agreed to the published version of the manuscript.

Funding: This research was funded by Shaanxi key industry innovation chain (Group) Foundation in social development field, China (2019ZDLSF02-05); Opening Foundation of Key Laboratory of Resource Biology and Biotechnology in Western China, Ministry of Education, (ZSK2018010).

Acknowledgments: We are grateful to Shengyue Li for assistance in mutant screening and Northwest University Zebrafish Core Facility for assistance with fish culture.

Conflicts of Interest: The authors declare no conflict of interest.

\section{References}

1. Fox, C.S.; Coady, S.F.; Sorlie, P.F.; D'Agostino, R.B.; Pencina, M.F.; Vasan, J.B.; Meigs, D.; Levy, P.J.; Savage, P.J. Increasing cardiovascular disease burden due to diabetes mellitus: The Framingham Heart Study. Circulation 2007, 12, 1544-1550.

2. Dagenais, G.R.; Leong, D.P.; Rangarajan, S.; Lanas, F.; Lopez-Jaramillo, P.; Gupta, R.; Diaz, R.; Avezum, A.; Oliveira, G.B.F.; Wielgosz, A.; et al. Variations in common diseases, hospital admissions, and deaths in middle-aged adults in 21 countries from five continents (PURE): A prospective cohort study. Lancet 2020, 10226, 785-794.

3. Staudt, D.; Stainier, D. Uncovering the molecular and cellular mechanisms of heart development using the zebrafish. Annu. Rev. Genet. 2012, 46, 397-418.

4. Zakaria, Z.Z.; Benslimane, F.M.; Nasrallah, G.K.; Shurbaji, S.; Younes, N.N.; Mraiche, F.; Da'as, S.I.; Yalcin, H.C. Using Zebrafish for Investigating the Molecular Mechanisms of Drug-Induced Cardiotoxicity. Biomed. Res. Int. 2018, 2018, 1642684 .

5. Nguyen, C.T.; Lu, Q.; Wang, Y.; Chen, J.N. Zebrafish as a model for cardiovascular development and disease. Drug Discov. Today Dis. Models. 2008, 5, 135-140. 
6. Korzh, S.; Pan, X.M.; Garcia-Lecea, C.L.; Winata, C.X.; Pan, X.T.; Wohland, T.V.; Korzh, V.Z.; Gong, Z. Requirement of vasculogenesis and blood circulation in late stages of liver growth in zebrafish. BMC Dev. Biol. 2008, 16, 8-84.

7. Brönnimann, D.; Annese, T.; Gorr, T.A.; Djonov, V. Splitting of circulating red blood cells as an in vivo mechanism of erythrocyte maturation in developing zebrafish, chick and mouse embryos. J. Exp. Biol. 2018, 221 , jeb184564.

8. Jagadeeswaran, P.; Sheehan, J.P.; Craig, F.E.; Troyer, D. Identification and characterization of zebrafish thrombocytes. Br. J. Haematol. 1999, 107, 731-738.

9. Sheehan, J.; Templer, M.; Gregory, M.; Hanumanthaiah, R.; Troyer, D.T.; Phan, T.B.; Thankavel, B.P.; Jagadeeswaran, P. Demonstration of the extrinsic coagulation pathway in teleostei: Identification of zebrafish coagulation factor VII. Proc. Natl. Acad. Sci. USA 2001, 98, 8768-8773.

10. Parng, C.; Seng, W.C.; Semino, C.P.; McGrath, P. Zebrafish: A preclinical model for drug screening. Assay Drug Dev. Technol. 2002, 1, 41-48.

11. Zhu, X.Y.; Liu, H.C.; Guo, S.Y.; Xia, B.; Song, R.S.; Lao, Q.C.; Xuan, Y.X.; Li, C.Q. A Zebrafish Thrombosis Model for Assessing Antithrombotic Drugs. Zebrafish 2016, 13, 335-344. [PubMed]

12. Lee, I.J.; Yang, Y.C.; Hsu, J.W.; Chang, W.T.; Chuang, Y.J.; Liau, I. Zebrafish model of photochemical thrombosis for translational research and thrombolytic screening in vivo. J. Biophotonics 2017, 10, 494-502. [PubMed]

13. Qi, Y.; Zhao, X.; Liu, H.; Wang, Y.; Zhao, C.; Zhao, T.; Zhao, B.; Wang, Y. Identification of a Quality Marker (Q-Marker) of Danhong Injection by the Zebrafish Thrombosis Model. J. Biophotonics 2017, 10, 494-502.

14. Zaidi, S.; Brueckner, M. Genetics and Genomics of Congenital Heart Disease. Circ. Res. 2017, 120, $923-940$.

15. Mably, J.D.; Mohideen, C.G.; Burns, J.N.; Chen, M.C.; Fishman, M.C. Heart of glass regulates the concentric growth of the heart in zebrafish. Curr. Biol. 2003, 13, 2138-2147.

16. Donat, S.; Lourenço, M.; Paolini, A.; Otten, C.; Renz, M.; Abdelilah-Seyfried, S. Heg1 and Ccm1/2 proteins control endocardial mechanosensitivity during zebrafish valvulogenesis. Elife 2018, 7, e28939.

17. Kleaveland, B.; Zheng, X.; Liu, J.J.; Blum, Y.; Tung, J.J.; Zou, Z.; Sweeney, S.M.; Chen, M.; Guo, L.; Lu, M.M.; et al. Regulation of cardiovascular development and integrity by the heart of glass-cerebral cavernous malformation protein pathway. Nat. Med. 2009, 15, 169-176.

18. Monaco, A.P.; Neve, R.L.; Colletti-Feener, C.; Bertelson, C.J.; Kurnit, D.M.; Kunkel, L.M. Isolation of candidate cDNAs for portions of the Duchenne muscular dystrophy gene. Nature 1986, 323, 646-650.

19. Kawahara, G.; Karpf, J.A.; Myers, J.A.; Alexander, M.S.; Guyon, J.R.; Kunkel, L.M. Drug screening in a zebrafish model of Duchenne muscular dystrophy. Proc. Natl. Acad. Sci. USA 2011, 108, 5331-5336.

20. Baraban, S.C.; Dinday, M.T.; Hortopan, G.A. Drug screening in Scn1a zebrafish mutant identifies clemizole as a potential Dravet syndrome treatment. Nat. Commun. 2013, 4, 2410.

21. Westerfield, M. The Zebrafish Book: Guide for the Laboratory Use of Zebrafish (Danio Rerio); University of Oregon Press: Eugene, UO, USA, 2000.

22. Kimmel, C.B.; Ballard, S.R.; Kimmel, S.B.; Ullmann, B.; Schilling, T.F. Stages of embryonic development of the zebrafish. Dev. Dyn. 1995, 203, 253-310. [CrossRef]

23. Grimes, A.C.; Erwin, K.N.; Stadt, H.A.; Hunter, G.L.; Gefroh, H.A.; Tsai, H.J.; Kirby, M.L. PCB126 exposure disrupts zebrafish ventricular and branchial but not early neural crest development. Toxicol. Sci. 2008, 106, 193-205. [CrossRef]

24. Gkatzis, K.; Thalgott, J.; Dos, D.; Martin, S.; Lamandé, N.; Carette, M.F.; Disch, F.; Snijder, R.J. Interaction Between ALK1 Signaling and Connexin40 in the Development of Arteriovenous Malformations. Arterioscler. Thromb. Vasc. Biol. 2016, 36, 707-717. [CrossRef]

25. Burns, C.G.; MacRae, C.A. Purification of hearts from zebrafish embryos. Biotechniques 2006, 40, $274-278$.

26. Mali, P.; Yang, L.; Esvelt, K.M.; Aach, J.; Guell, M.; DiCarlo, J.E.; Norville, J.E.; Church, G.M. RNA-guided human genome engineering via Cas9. Science 2013, 339, 823-826. [CrossRef]

27. Liu, C.X.; Li, C.Y.; Hu, C.C.; Wang, Y.; Lin, J.; Jiang, Y.H.; Li, Q.; Xu, X. CRISPR/Cas9-induced shank3b mutant zebrafish display autism-like behaviors. Mol. Autism. 2018, 9, 23. [CrossRef]

28. Moreno-Mateos, M.A.; Vejnar, C.E.; Beaudoin, J.D.; Fernandez, J.P.; Mis, E.K.; Khokha, M.K.; Giraldez, A.J. CRISPRscan: Designing highly efficient sgRNAs for CRISPR-Cas9 targeting in vivo. Nat. Methods 2015, 12, 982-988. [CrossRef] 
29. Tian, J.; Shao, J.; Liu, C.; Hou, H.Y.; Chou, C.W.; Shboul, M.; Li, G.Q.; El-Khateeb, M.; Samarah, O.Q.; Kou, Y.; et al. Deficiency of lrp4 in zebrafish and human LRP4 mutation induce aberrant activation of Jagged-Notch signaling in fin and limb development. Cell. Mol. Life Sci. 2019, 76, 163-178. [CrossRef]

30. Tian, J.; Ling, L.; Shboul, M.; Lee, H.; O'Connor, B.; Merriman, B.; Nelson, S.F.; Cool, S.; Ababneh, O.H.; Al-Hadidy, A.; et al. Loss of CHSY1, a secreted FRINGE enzyme, causes syndromic brachydactyly in humans via increased NOTCH signaling. Am. J. Hum. Genet. 2010, 87, 768-778. [CrossRef]

31. Bhakta, M.; Padanad, M.S.; Harris, J.P.; Lubczyk, C.; Amatruda, J.F.; Munshi, N.A.-O.X. pouC Regulates Expression of bmp4 During Atrioventricular Canal Formation in Zebrafish. Dev. Dyn. 2019, 248, 173-188. [CrossRef]

32. Korzh, S.; Winata, C.L.; Zheng, W.; Yang, S.; Yin, A.; Ingham, P.; Korzh, V.; Gong, Z. The interaction of epithelial Ihha and mesenchymal Fgf10 in zebrafish esophageal and swimbladder development. Dev. Biol. 2011, 359, 262-276. [CrossRef] [PubMed]

33. Huang, C.C.; Lawson, N.D.; Weinstein, B.M.; Johnson, S.L. reg6 is required for branching morphogenesis during blood vessel regeneration in zebrafish caudal fins. Dev. Biol. 2003, 264, 263-274. [CrossRef] [PubMed]

34. Du, L.; Xu, J.; Li, X.; Ma, N.; Liu, Y.; Peng, J.; Osato, M.; Zhang, W.; Wen, Z. Rumba and Haus3 are essential factors for the maintenance of hematopoietic stem/progenitor cells during zebrafish hematopoiesis. Development 2011, 138, 619-629. [CrossRef]

35. Hu, G.; Mahady, G.B.; Li, S.; Hoi, M.P.; Wang, Y.H.; Lee, S.M. Polysaccharides from astragali radix restore chemical-induced blood vessel loss in zebrafish. Vasc. Cell 2012, 4, 2. [CrossRef]

36. Tse, H.Y.; Hui, M.N.; Li, L.; Lee, S.M.; Leung, A.Y.; Cheng, S.H. Angiogenic efficacy of simplified 2-herb formula (NF3) in zebrafish embryos in vivo and rat aortic ring in vitro. J. Ethnopharmacol. 2012, 139, 447-453. [CrossRef]

37. Adams, J.D.; Wang, R.; Yang, J.; Lien, E.J. Preclinical and clinical examinations of Salvia miltiorrhiza and its tanshinones in ischemic conditions. Chin. Med. 2006, 23, 3. [CrossRef]

38. Buchwald, A.B.; Hammerschmidt, S.; Stevens, J.; Göring, J.; Nebendahl, K.; Unterberg, C. Inhibition of neointimal proliferation after coronary angioplasty by low-molecular-weight heparin (clivarine) and polyethyleneglycol-hirudin. J. Cardiovasc. Pharmacol. 1996, 28, 481-487. [CrossRef]

39. Shedoeva, A.; Leavesley, D.; Upton, Z.; Fan, C. Wound Healing and the Use of Medicinal Plants. Evid. Based Complement. Alternat. Med. 2019, 2019, 2684108. [CrossRef]

40. Wang, J.; Xiong, X.; Feng, B. Cardiovascular effects of salvianolic Acid B. Evid. Based Complement. Alternat. Med. 2013, 2013, 247948. [CrossRef]

41. Li, W.; Zhi, W.; Liu, F.; Zhao, J.; Yao, Q.; Niu, X. Paeoniflorin inhibits VSMCs proliferation and migration by arresting cell cycle and activating HO-1 through MAPKs and NF-kB pathway. Int. Immunopharmacol. 2018, 54, 103-111. [CrossRef]

42. Chen, H.; Dong, Y.; He, X.; Li, J.; Wang, J. Paeoniflorin improves cardiac function and decreases adverse postinfarction left ventricular remodeling in a rat model of acute myocardial infarction. Drug Des. Devel. Ther. 2018, 12, 823-836. [CrossRef] [PubMed]

43. Alam, M.A.; Sernia, C.; Brown, L. Ferulic acid improves cardiovascular and kidney structure and function in hypertensive rats. J. Cardiovasc. Pharmacol. 2013, 61, 240-249. [CrossRef] [PubMed]

44. Chowdhury, S.; Ghosh, S.; Rashid, K.; Sil, P.C. Deciphering the role of ferulic acid against streptozotocin-induced cellular stress in the cardiac tissue of diabetic rats. Food Chem. Toxicol. 2016, 97, 187-198. [CrossRef] [PubMed]

45. Liu, C.; Huang, Y. Chinese Herbal Medicine on Cardiovascular Diseases and the Mechanisms of Action. Front. Pharmacol. 2016, 7, 469. [CrossRef] [PubMed]

46. Fu, S.; Zhang, J.; Menniti-Ippolito, F.; Gao, X.; Galeotti, F.; Massari, M.; Hu, L.; Zhang, B.; Ferrelli, R.; Fauci, A.; et al. Huangqi injection (a traditional Chinese patent medicine) for chronic heart failure: A systematic review. PLOS ONE 2011, 6, e19604. [CrossRef]

47. Ma, X.; Zhang, K.; Li, H.; Han, S.; Ma, Z.; Tu, P. Extracts from Astragalus membranaceus limit myocardial cell death and improve cardiac function in a rat model of myocardial ischemia. J. Ethnopharmacol. 2013, 149, 720-728. [CrossRef]

48. Ding, M.; Ye, T.X.; Zhao, G.R.; Yuan, Y.J.; Guo, Z.X. Aqueous extract of Salvia miltiorrhiza attenuates increased endothelial permeability induced by tumor necrosis factor-alpha. Int. Immunopharmacol. 2005, 5, 1641-1651. [CrossRef] 
49. Wang, X.; Wang, Y.; Jiang, M.; Zhu, Y.; Hu, L.; Fan, G.; Wang, Y.; Li, X.; Gao, X. Differential cardioprotective effects of salvianolic acid and tanshinone on acute myocardial infarction are mediated by unique signaling pathways. J. Ethnopharmacol. 2011, 135, 662-671. [CrossRef]

50. Ren, J.; Fu, L.; Nile, S.H.; Zhang, J.; Kai, G. Salvia miltiorrhiza in Treating Cardiovascular Diseases: A Review on Its Pharmacological and Clinical Applications. Front. Pharmacol. 2019, 10, 753. [CrossRef]

51. Liu, J.F.; Hu, A.N.; Zan, J.F.; Wang, P.; You, Q.Y.; Tan, A.H. Network Pharmacology Deciphering Mechanisms of Volatiles of Wendan Granule for the Treatment of Alzheimer's Disease. Evid. Based Complement. Alternat. Med. 2019, 2018, 7826769. [CrossRef]

52. Li, L.; Zhou, X.; Li, N.; Sun, M.; Lv, J.; Xu, Z. Herbal drugs against cardiovascular disease: Traditional medicine and modern development. Drug Discov. Today 2015, 20, 1074-1086. [CrossRef] [PubMed]

53. Gu, G.; Na, Y.; Chung, H.; Seok, S.H.; Lee, H.Y. Zebrafish Larvae Model of Dilated Cardiomyopathy Induced by Terfenadine. Korean Circ. J. 2017, 47, 960-969. [CrossRef] [PubMed]

Publisher's Note: MDPI stays neutral with regard to jurisdictional claims in published maps and institutional affiliations.

(C) 2020 by the authors. Licensee MDPI, Basel, Switzerland. This article is an open access article distributed under the terms and conditions of the Creative Commons Attribution (CC BY) license (http://creativecommons.org/licenses/by/4.0/). 